\title{
Psychosocial predictors of posttreatment pain after nonmetastatic breast cancer treatment: a systematic review and meta-analysis of prospective studies
}

This article was published in the following Dove Press journal:

Journal of Pain Research

\author{
M Johannsen ${ }^{1,2}$ \\ Y Frederiksen ${ }^{1,2}$ \\ $A B$ Jensen $^{3}$ \\ R Zachariae ${ }^{1,2}$ \\ 'Unit for Psychoncology and \\ Health Psychology, Department of \\ Oncology, Aarhus University Hospital, \\ 2Department of Psychology, Aarhus \\ University, ${ }^{3}$ Department of Oncology, \\ Aarhus University Hospital, Aarhus C, \\ Denmark
}

\begin{abstract}
Background: The search for risk factors of pain after breast cancer, which affects a considerable proportion of the women, has primarily focused on clinical factors. The aim of this meta-analysis was to explore the less well-studied psychosocial predictors of pain after breast cancer treatment. Methods: Two independent searches were conducted in PubMed, PsycINFO, Web of Science, and CINAHL. Eligible studies were prospective, observational studies of women aged $\geq 18$ years, diagnosed and treated for nonmetastatic breast cancer $\geq 3$ months previously. Additional inclusion criteria were that studies had assessed at least one pain outcome and at least one psychosocial predictor. The psychosocial predictors investigated included: 1) psychological-behavioral states, 2) psychological traits, and 3) social support. Effect size correlations (ESr) were chosen as the effect size and pooled using a random effects model. We also explored a number of study characteristics as possible moderators of the effect with meta-regression.
\end{abstract}

Results: Of the total of 13 eligible studies identified, most studies measured psychosocial predictors at presurgery. Neither psychological-behavioral states (ESr: $0.05 ; p=0.13 ; \mathrm{K}=11$ ) nor psychological traits (ESr: $0.02 ; p=0.48 ; \mathrm{K}=6$ ) emerged as statistically significant predictors of pain. In contrast, higher levels of social support were statistically significantly associated with less pain (ESr: $-0.24 ; p<0.001 ; \mathrm{K}=4$ ). In studies of psychological-behavioral states, longer follow-up was associated with smaller effect sizes $(p=0.023)$. Furthermore, older mean sample age was associated with larger effect sizes for both psychological-behavioral states $(p=0.0004)$ and psychological traits $(p=0.035)$.

Conclusion: The results of this meta-analysis suggest that psychosocial factors measured at presurgery may only be of modest predictive value in identifying women at risk of developing pain after breast cancer treatment. While speculative, psychosocial factors may play a larger role in the postsurgery trajectory, which could be valuable to investigate in future studies.

Keywords: breast cancer, pain, predictors, personality traits, social support, behavioral symptoms

\section{Introduction}

Pain is a prevalent late effect after breast cancer treatment, affecting $16 \%-20 \%$ of women up to several years after completed primary treatment. ${ }^{1-3}$ Pain is defined as persistent when lasting $\geq 3-6$ months after surgery. ${ }^{4,5}$ Given the high prevalence of persistent pain after breast cancer treatment and the negative impact of pain on quality of life, ${ }^{6,7}$ several studies have investigated a range of possible risk factors for the development of posttreatment pain in breast cancer survivors. So far, most research has focused on
Department of Oncology and Psychology, Unit for Psycho-Oncology and Health Psychology, Aarhus University Hospital, Aarhus University, Bartolins Allé 9, Building 1340, DK-8000 Aarhus C, Denmark

Tel +4587165956

Email majajo@psy.au.dk 
clinical and demographic factors, with the available evidence suggesting that more invasive axillary surgery, radiotherapy, endocrine treatment for postmenopausal women, higher body mass index, preoperative pain, and younger age are relatively consistent predictors of posttreatment pain. . $^{2,3,5,8-10}$

Based on the advances in pain research, the pain experienced by breast cancer survivors may, however, not be fully explained by these factors. ${ }^{11}$ Historically, the predominant biomedical paradigm of pain as primarily a peripheral sensory process was transformed by the introduction of "the gatecontrol theory" of pain in $1960 \mathrm{~s},{ }^{12}$ revealing the dynamic role of the brain in the interpretative process of the pain response and leading the International Association for the Study of Pain to define pain as a complex bio-psycho-social phenomenon. ${ }^{4}$ In further theoretical developments, pain is construed as involving a "neural matrix", ${ }^{13}$ including both sensory and nociceptive circuitries, modulation by inhibitory and facilitatory centers, and input from cognitive and emotional centers. ${ }^{14}$ Taken together, psychosocial factors are thus assumed to account for a considerable proportion of the individual differences in the response to both acute and chronic pain. ${ }^{14,15}$ Identification of possible psychosocial risk factors could offer clinically valuable information both in pretreatment pain risk assessment and in posttreatment rehabilitation efforts.

At present, the available results are inconsistent, with some studies finding associations between psychosocial factors (eg, pain catastrophizing, ${ }^{16}$ depression, ${ }^{16-19}$ anxiety, ${ }^{19-21}$ and distress ${ }^{9}$ ) and persistent pain after breast cancer surgery, while others fail to find such associations. ${ }^{22-25}$ This could partially be explained by the considerable methodological heterogeneity of the available research, for example, in the pain and psychosocial measures used, the timing of assessments, sample sizes, and analytical strategies (whether analyses have adjusted for other variables or not). Furthermore, the majority of studies appear to have used cross-sectional designs, thereby limiting the ability to determine temporal precedence of the risk factor in relation to pain (cause vs consequence). So far, two narrative systematic reviews have examined the role of psychosocial factors. ${ }^{5,14}$ The authors of the first review published in 2011 concluded that while the evidence suggests associations between psychosocial factors and pain in women treated for breast cancer, the causal direction remained unclear due to few available prospective studies. ${ }^{5}$ The second review, also including studies of nonbreast cancer patients, published in 2014, concluded that both retrospective and prospective studies suggested that pre-existing psychological symptoms predict pain after breast cancer treatment. ${ }^{14}$ However, as the evidence reviewed was not restricted to breast cancer patients, the generalizability of the findings to breast cancer patients may be limited.

So far, there have been no meta-analytical attempts to quantify the existing evidence on psychosocial risk factors of pain after breast cancer treatment. Our aim was, therefore, to conduct a systematic review of the available prospective studies on psychosocial predictors of posttreatment pain in women treated for nonmetastatic breast cancer, combine the results with meta-analysis, and explore study characteristics as possible moderators of the associations.

\section{Materials and methods}

This review was preregistered with PROSPERO (Johannsen et al, 2017; registration no.: CRD42017062218) and conducted in accordance with the Preferred Reporting Items for Systematic Reviews and Meta-Analyses criteria. ${ }^{26}$

\section{Data sources and search strategy}

The electronic databases PubMed, PsycINFO, Web of Science, and CINAHL were searched independently by two authors (MJ, YF) for relevant records published until June 2017. Based on the population, indicator, comparator, outcome (PICO) approach, ${ }^{27}$ the final search string consisted of combinations of subject headings (MeSH terms) and keywords referring to population ("breast neopl*" OR "breast cancer" OR "breast carcinoma" OR "breast tumor" OR "mammary cancer"), indicator ("predict*" OR "risk factor*" OR "relative risk" OR "odds ratio" OR logistic OR regression), and outcome (pain OR "post mastectomy pain syndrome" OR "persistent post-surgical pain" OR ache OR “chronic post-surgical pain"), as shown in Table S1. No keywords were included for comparator. In addition, a backward search (snowballing) was conducted of reference lists of identified articles, together with a forward search (citation tracking) to identify additional relevant publications.

\section{Selection procedure}

The review included observational studies of adult women ( $\geq 18$ years) treated for nonmetastatic breast cancer, which had assessed pain as an outcome variable with a relevant, validated instrument. Only studies including at least one quantitative measure of a possible psychosocial risk factor were included. In this review, psychosocial risk factors were operationalized as 1) psychological-behavioral states (eg, depressive symptoms, pain catastrophizing), 2) psychological traits (general stable tendencies, eg, personality traits, locus of control), and 3) social support (eg, practical support, emotional support). We did not include marital status as a 
measure of social support, but regard it a demographic factor, as marital status does not indicate the quality of the relationship (if married) or lack of social support from sources (if single). We excluded studies of men treated for breast cancer, women with metastatic breast cancer, and women having received surgery $<3$ months previously. Studies focusing on pain-related outcomes such as sensory disturbances, shoulder morbidity, and lymphedema, were also excluded. Qualitative studies, intervention studies, case studies, and reviews were not considered eligible for inclusion. Post hoc, we implemented additional inclusion criteria by including only studies using prospective designs. This allowed us to increase the quality of the included studies in terms of establishing temporal precedence of the predictor in relation to pain. We did not include the gray literature (eg, dissertations, unpublished manuscripts), but assessed the risk of publication bias statistically (see the "Meta-analytical strategy" section). Three authors (MJ, YF, RZ) discussed disagreements until a negotiated conclusion was reached. Interrater reliability was assessed using Cohen's kappa. ${ }^{28,29}$

\section{Quality assessment}

All studies included were subjected to a study quality assessment by two independent raters (MJ, YF). We used a 14-item quality assessment tool developed specifically for observational studies. ${ }^{30}$ We modified the tool by 1 ) renaming "the exposure variable" as "predictor variable", 2) excluding three criteria (items $8,10,12$ ) that were not relevant in our review, and 3 ) adding two criteria relating to adequate reporting of the studies, namely, whether the studies included a study flow diagram and provided descriptive data on key clinical sample characteristics (eg, proportion receiving chemotherapy), yielding a final total of 13 items. Each quality criterion was assigned 1 point, yielding a total study quality score of $0-13$, with higher scores indicating higher study quality. Again, disagreements between the raters (MJ, YF) were discussed with a third author (RZ) until a conclusion was reached. Interrater reliability was assessed using Cohen's kappa. ${ }^{28,29}$

\section{Meta-analytical strategy}

First, effect sizes representing the magnitude of the association between the independent predictor (ie, psychosocial factor) and the dependent variable (ie, pain) were calculated for each study. The effect size correlation $(\mathrm{ESr})^{31}$ was chosen as the effect size. ESr is an equivalent of Pearson's $r$, with values ranging from -1.00 to +1.00 . In this study, negative values indicate an inverse relationship between the predictor and pain, that is, higher levels of the predictor being associated with lower levels of pain, while positive values indicate a positive association, with higher levels of the predictor being associated with higher levels of pain. If necessary, ESr was estimated from other reported data, for example, means and SDs, $\beta$-coefficients, and odds ratios, using various formulas from the literature. If an eligible study did not report data suitable for conversion, we contacted the authors. If studies reported both unadjusted and adjusted results for a given psychosocial predictor, we ensured independence of results in the analyses by selecting adjusted results over unadjusted results in the meta-analytic evaluation. If studies had included more than one follow-up assessment time point, data collected at the longest follow-up was chosen. The effect sizes of the individual studies were combined using a random effects model with inverse variance weighting giving studies with larger samples greater weight than studies with small sample sizes. We calculated three overall effect sizes for: 1) psychological-behavioral states, 2) psychological traits, and 3) social support. ESrs $<0.10, \geq 0.25$, and $\geq 0.40$ were considered small, medium, and large, respectively. ${ }^{32,33}$

Publication bias, a widespread problem when conducting meta-analyses, ${ }^{34}$ was evaluated with funnel plots, Egger's method, and by calculating fail-safe numbers. ${ }^{35-37} \mathrm{~A}$ funnel plot is a graphic illustration of study effect sizes (ESs) in relation to study size or precision. Egger's test provides a statistic for the skewness of results. ${ }^{35}$ Calculation of fail-safe numbers is aimed at achieving an indication of the number of unpublished studies with null findings that would reduce the result to statistical nonsignificance $(p>0.05)$. It has been suggested that a reasonable level is achieved if the fail-safe number exceeds $(5 \mathrm{~K}+10 ; \mathrm{K}=$ number of studies in the metaanalysis). ${ }^{35}$ If the results were suggestive of publication bias, an adjusted ES was calculated using Duval and Tweedie's trim and fill method, ${ }^{38}$ which imputes ESs of missing studies and recalculates the ES accordingly.

Heterogeneity was explored using Q and $I^{2}$ statistics. ${ }^{39}$ Heterogeneity tests are aimed at determining whether results reflect genuine differences (heterogeneity), or whether the variation is due to random error. Due to generally low statistical power of heterogeneity tests, a more liberal $p$ value of 0.10 was used to determine significant heterogeneity. ${ }^{40}$ The $I^{2}$ statistic estimates the amount of variance in a pooled effect size accounted for by heterogeneity, and is unaffected by the number of studies $(\mathrm{K}) .{ }^{41} I^{2}$ values of $0 \%, 25 \%, 50 \%$, and $75 \%$ are taken to indicate no, low, moderate, and high observed heterogeneity, respectively.

Finally, we conducted a number of moderator analyses. When more than three studies reported relevant data, we 
conducted between-study differences in ESs according to the type of analysis (adjusted vs unadjusted), specificity of the pain measure (specific vs generic), whether pain was primary outcome (yes vs no), baseline measurement of pain (presurgery vs postsurgery), and anxiety and depression instrument used. In addition, we investigated the possible influence of study quality, mean sample age, and mean follow-up time on the results with meta-regression.

\section{Results}

\section{Study selection}

The search of the electronic databases yielded 937 hits (PubMed: 278, PsycINFO: 138, Web of Science: 295, CINAHL: 226). After duplicate removal $(n=275)$, the total number of unique hits was 662 . In the first screening, all records were screened for eligibility based on title and abstract according to our inclusion and exclusion criteria, leading to the exclusion of 455 records (Figure 1). Full-text papers were retrieved for the remaining 207 records. In the second screening, 194 of these were excluded, yielding a final total of 13 independent studies to be included in this metaanalysis. ${ }^{18,19,21,23,42-49}$ Cohen's kappa between the two raters was 0.62 , corresponding to substantial level of agreement. ${ }^{29}$

\section{Study characteristics}

The characteristics of the included studies are shown in Table 1. The studies were published between 2006 and 2016, with the majority of studies from Europe $(\mathrm{K}=8)$ and fewer from the USA $(K=3)$ and Australia $(K=2)$. Pain was assessed by breast cancer-pain specific measures in eight studies, while the remaining five studies used generic pain measures only. Four studies used pain as a continuous variable in their analyses, whereas the remaining studies defined pain as a binary $(K=7)$ or ordinal variable $(K=3)$. One study ${ }^{25}$ reported pain both as a continuous and a categorical variable. Seven out of 13 studies conducted their baseline assessment prior to breast cancer surgery, with the remaining 5 studies establishing the baseline after surgery. All studies used selfreport measures to assess psychosocial risk factors, with 11 studies assessing psychological-behavioral states, including depressive symptoms $(\mathrm{K}=8 ; 3$ with the Hospital Anxiety and Depression Scale [HADS] and 5 with the Spielberger State-Trait Anxiety Inventory [STAI]); anxiety ( $\mathrm{K}=8$; 3 with HADS and 3 studies with the Beck Depression Inventory); overall psychological distress $(\mathrm{K}=3)$; pain catastrophizing $(\mathrm{K}=4)$; intrusion, avoidance, or emotional repression $(\mathrm{K}=3)$; and psychological, spiritual, or positive well-being $(\mathrm{K}=3)$. Six studies included measures of psychological traits, including trait anxiety $(\mathrm{K}=4)$, various personality traits $(\mathrm{K}=1)$, locus of control or self-control $(\mathrm{K}=2)$, psychological robustness $(\mathrm{K}=1)$, and life-orientation $(\mathrm{K}=1)$. Finally, four studies measured social support, including social functioning and wellbeing $(K=2)$ and multifaceted social support (ie, emotional support, tangible social support, positive social interaction) $(\mathrm{K}=2)$. Sample sizes ranged from 82 to 1683 (mean $\mathrm{n}=421$, $\mathrm{SD}=454$ ), yielding a total sample of 5457. Mean sample age ranged from 54 to 62 years (mean $=57.5$ years, $\mathrm{SD}=2.85$ ). Ten out of 13 studies included more than one follow-up (range: 3-60 months), with a mean time to follow-up of 16.3 months $(\mathrm{SD}=15.71)$.

\section{Study quality}

The study quality scores of the included studies are presented in Table 2. They ranged from 7 to 13 , with a mean score of 10.4 ( $\mathrm{SD}=2.3)$. Interrater reliabilities (Cohen's kappa) for the individual study quality indicators ranged from 0.44 to 1.00 , corresponding to fair to almost perfect agreement. ${ }^{28}$

\section{Meta-analysis}

Eleven studies of a total of 2176 women were included in the analyses of the psychological-behavioral states. The results indicated a positive, but negligible $(\mathrm{ESr}=0.05)$ and statistically nonsignificant ( $p=0.13$ ) association between psychologicalbehavioral states and subsequent pain (Table 3 ). In a post hoc analysis, we pooled the effect sizes for anxiety $(\mathrm{K}=8)$ and depression $(\mathrm{K}=8)$ separately. Neither reached statistical significance. The results concerning the association between psychological traits and pain $(\mathrm{K}=6 ; \mathrm{n}=1449)$ also failed to show a statistically significant relationship ( $\mathrm{ESr}=0.02$; $p=0.48)$. In contrast, the pooled results for social support $(\mathrm{K}=4 ; \mathrm{n}=1057)$ showed a statistically significant negative association $(\mathrm{ESr}=-0.24, p<0.001)$ between social support and pain corresponding to a small to medium effect size. Our analyses indicated the possibility of publication bias for social support, and we subsequently imputed missing studies yielding a slightly larger effect size $(\mathrm{ESr}=-0.25)$. Examining the robustness of the results for social support, the fail-safe number of 49 was larger than the criterion of 30 , indicating a robust result. There were no indications of publication bias for psychological-behavioral states or psychological traits. The effect sizes found for social support did not appear heterogeneous $\left(I^{2}=0, p=0.42\right)$. In contrast, substantial heterogeneity was indicated for both psychological-behavioral states $\left(I^{2}=72.8, p>0.001\right)$ and psychological traits $\left(I^{2}=71, p=0.004\right)$. 


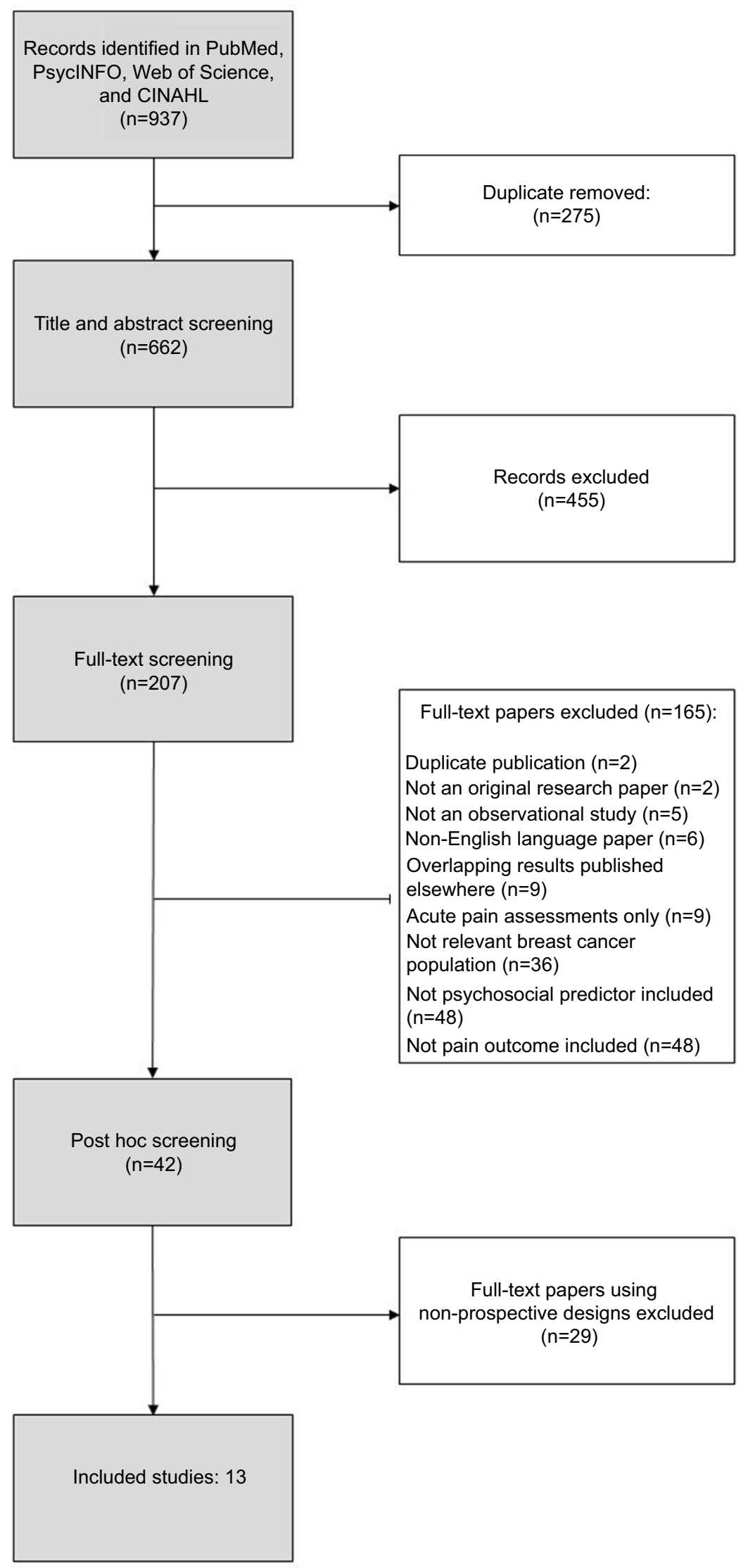

Figure I Flow diagram of study selection. 


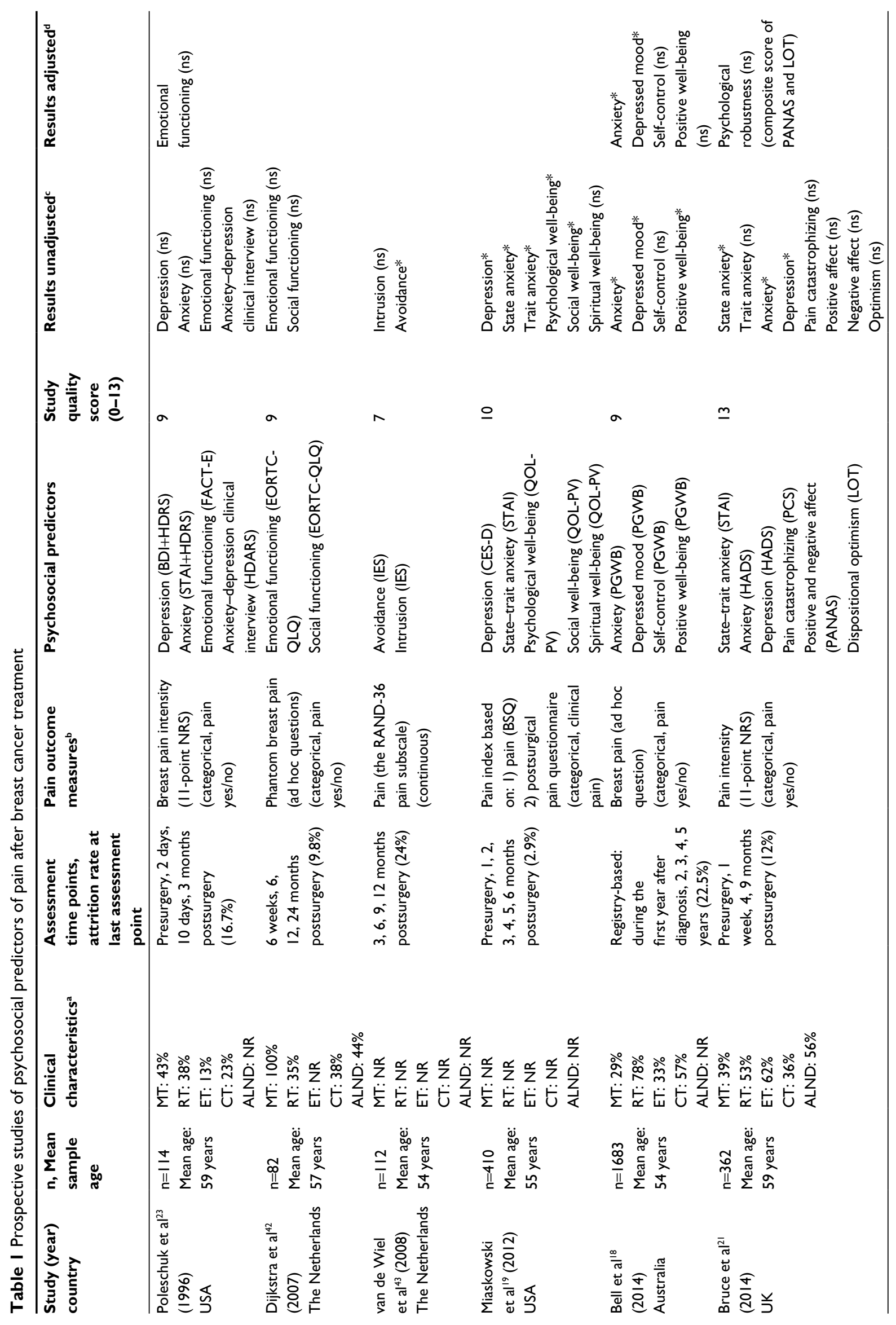




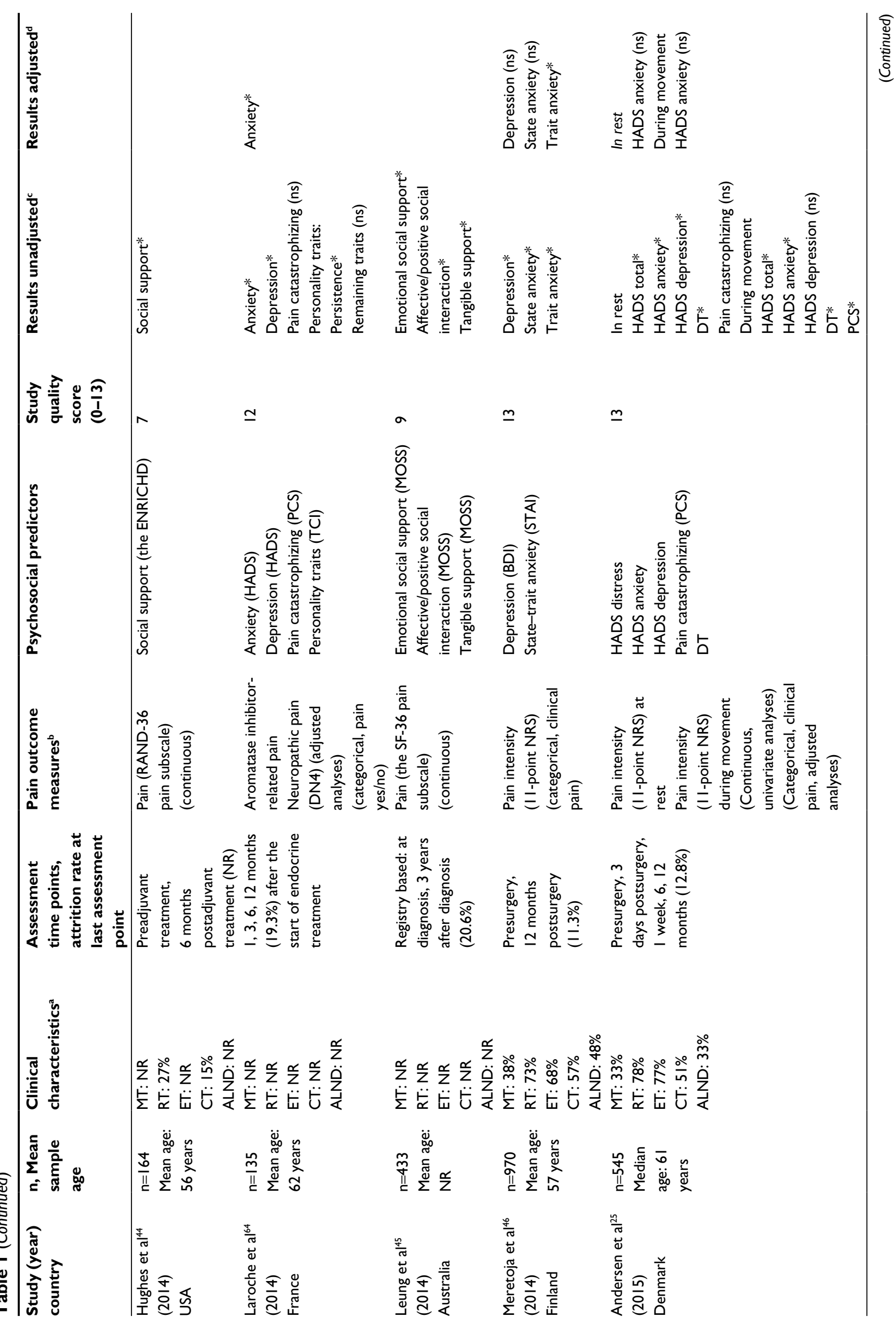




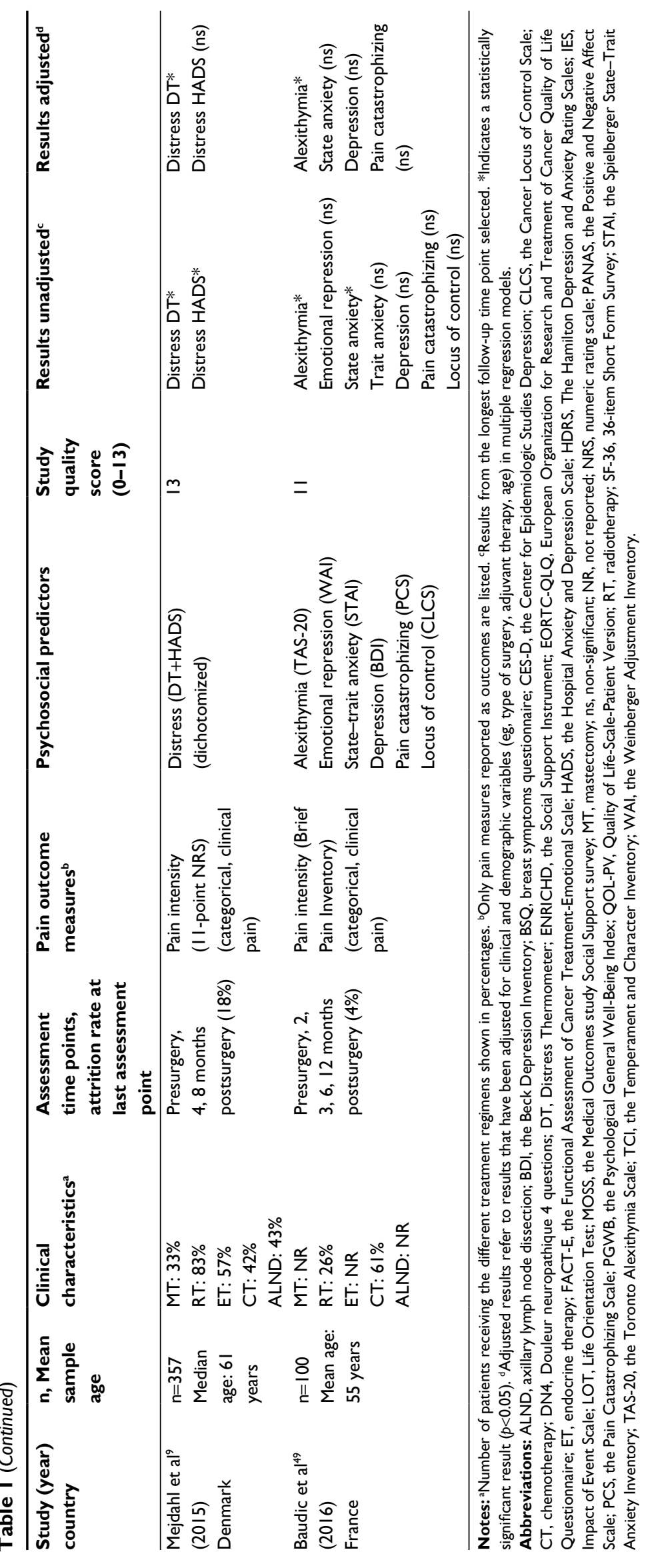


Table 2 Quality assessment of included studies $(K=13)^{\mathrm{a}}$

\begin{tabular}{|c|c|c|c|c|c|c|c|c|c|c|c|c|c|c|}
\hline Study & $\mathbf{I}$ & 2 & 3 & 4 & 5 & 6 & 7 & 8 & 9 & 10 & II & 12 & 13 & Tota \\
\hline I. Poleschuk et $\mathrm{al}^{23}$ & 1 & $\mathrm{I}$ & $\mathrm{I}$ & $\mathrm{I}$ & $\mathrm{I}$ & $\mathrm{I}$ & 0 & 1 & 0 & 1 & $0^{\mathrm{b}}$ & 0 & 1 & 9 \\
\hline 2. Dijkstra et $\mathrm{al}^{42}$ & I & 1 & 0 & I & 1 & I & I & I & 0 & I & 0 & 0 & I & 9 \\
\hline 3. van de Wiel et $\mathrm{a}^{43}$ & I & I & I & I & 1 & 0 & I & I & 0 & 0 & 0 & 0 & 0 & 7 \\
\hline 4. Miaskowski et al ${ }^{19}$ & I & 1 & I & I & 1 & I & I & I & I & I & 0 & 0 & 0 & 10 \\
\hline 5. Bell et $\mathrm{al}^{18}$ & I & 1 & I & I & 1 & 0 & I & I & 0 & 0 & I & 0 & 1 & 9 \\
\hline 6. Bruce et $\mathrm{al}^{21}$ & I & 1 & I & I & 1 & I & I & I & I & I & I & I & I & 13 \\
\hline 7. Hughes et $\mathrm{al}^{44}$ & I & 1 & 0 & I & 1 & I & I & I & 0 & 0 & 0 & 0 & 0 & 7 \\
\hline 8. Laroche et $\mathrm{a}^{64}$ & I & 1 & I & I & I & I & I & I & I & I & I & I & 0 & 12 \\
\hline 9. Leung et $\mathrm{al}^{45}$ & I & 1 & I & I & 1 & I & I & I & 0 & 0 & I & 0 & 0 & 9 \\
\hline I0. Meretoja et al ${ }^{46}$ & I & I & I & I & I & I & I & I & I & I & I & I & I & 13 \\
\hline II. Andersen et al ${ }^{47}$ & I & I & I & I & 1 & I & I & I & I & I & I & I & I & 13 \\
\hline I2. Mejdahl et $\mathrm{al}^{48}$ & 1 & 1 & I & I & 1 & I & I & I & I & I & 1 & I & I & 13 \\
\hline 13. Baudic et $\left.\mathrm{a}\right|^{49}$ & $\mathrm{I}$ & $\mathrm{I}$ & $\mathrm{I}$ & $\mathrm{I}$ & $\mathrm{I}$ & 1 & I & $\mathrm{I}$ & I & I & I & 0 & 0 & 11 \\
\hline Total & 13 & 13 & 11 & 13 & 13 & II & 12 & 13 & 7 & 9 & 8 & 6 & 7 & 10 \\
\hline
\end{tabular}

Notes: aDescription of quality indicators: I: Was the research question or objective clearly stated? 2: Was the study population clearly specified and defined? 3: Was the participation rate of all eligible patients $\geq 50 \%$ ? 4 : Were the subjects recruited from same or similar populations with inclusion/exclusion criteria prespecified and applied uniformly? 5: Was a sample justification, power description, or variance and effect estimates provided? 6: Were the predictor(s) of interest measured prior to the outcome(s) being measured? 7: Was the time period sufficient, so that one could reasonably expect to see an association between exposure and outcome if it existed? 8: Were the predictor measures (independent variables) clearly defined, valid, reliable, and implemented consistently across all study participants? 9: Were the outcome measures (dependent variables) clearly defined, valid, reliable, and implemented? I0: Was loss to follow-up after baseline $20 \%$ or less? II: Were key potential confounding variables measured and adjusted statistically for their impact on the relationship between the predictor(s) and outcome(s)? 12: Was a study diagram over the study participants

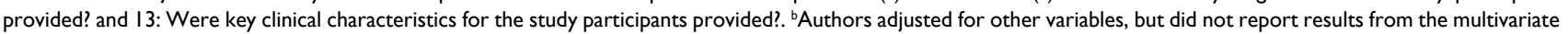
analysis that could be used in this meta-analysis (authors were contacted, but the data were no longer available).

\section{Moderator analyses}

Possible sources of between-study differences were explored with subgroup analyses, but only for psychological-behavioral states due to the insufficient number of studies of psychological traits and social support (Table 4). The pooled effect sizes were generally larger for studies using generic pain measures $(\mathrm{ESr}=0.10)$ compared with breast cancer-specific measures (0.04), for unadjusted analyses (0.09) compared with adjusted analyses (0.03), for studies with a baseline assessment at presurgery (0.08) compared with studies with a baseline assessment postsurgery (0.05), and for studies using the HADS depression subscale (0.12) compared with studies using the Beck Depression Inventory (0.03). None of the results, however, reached statistical significance. In addition, the possible moderating influence of the continuous variables of study quality, mean sample age, and time to follow-up was explored with meta-regression for psychological-behavioral states and psychological traits. Social support was not examined due to the small number of studies. Older age was statistically significantly associated with larger associations between psychological-behavioral states and pain $(p=0.023)$, and longer time to follow-up was associated with smaller associations between both psychological-behavioral states $(p=0.0004)$ and psychological traits ( $p=0.035$ ) and pain after breast cancer treatment. None of the remaining meta-regressions reached statistical significance.

\section{Discussion}

The majority of studies and reviews have mainly focused on the biomedical predictors of pain in breast cancer survivors (eg, Andersen and Kehlet, ${ }^{5}$ Wang et al, ${ }^{8}$ Nijs et a ${ }^{50}$ ) and, so far, only two systematic reviews have examined the role of psychosocial factors, ${ }^{5,14}$ neither of which included prospective studies only or subjected the available data to metaanalytical evaluation. Thus, to our knowledge, our study is the first meta-analysis of prospective studies of breast cancer survivors. Our results suggest that overall, the predominantly presurgically assessed psychological states and traits do not appear to predict pain after breast cancer.

In contrast, a statistically significant prospective association was found between social support and pain, with higher levels of social support predicting lower levels of posttreatment pain. One possible interpretation could be that women experiencing higher levels of support might receive more practical support (eg, with daily chores), thereby reducing the risk of straining the arm/shoulder. Another could be that higher levels of social support might promote the use of more adaptive pain coping strategies. ${ }^{51,52}$ However, the few available prospective studies did not explore the association between pain and different types of social support, and the suggested explanations remain speculative. Furthermore, although our results appeared robust when considering the possibility of publication bias, two out of four studies 
Table 3 Pooled effect sizes of included studies $(K=13)$ and between-group differences

\begin{tabular}{|c|c|c|c|c|c|c|c|c|c|c|c|}
\hline \multirow[t]{2}{*}{ Main predictors } & \multicolumn{2}{|c|}{$\begin{array}{l}\text { Sample } \\
\text { size }\end{array}$} & \multicolumn{4}{|c|}{ Heterogeneity $^{\mathbf{a}}$} & \multicolumn{3}{|c|}{ Pooled effect sizes } & \multirow{2}{*}{$\begin{array}{l}\text { Fail-safe } \\
n^{b}\end{array}$} & \multirow{2}{*}{$\begin{array}{l}\text { Fail-safe } \\
\text { criterion }\end{array}$} \\
\hline & $\overline{\mathbf{K}^{\mathbf{d}}}$ & $n^{d}$ & $\mathbf{Q}$ & $d f$ & p-value & $I^{2}$ & ESre & $95 \% \mathrm{Cl}$ & $p$-value & & \\
\hline \multicolumn{12}{|l|}{ Overall effects } \\
\hline Psychological-behavioral states & 11 & 2176 & 36.7 & 10 & $<0.001$ & 72.8 & 0.05 & $-0.01-0.11$ & 0.132 & & \\
\hline Psychological traits & 6 & 1449 & 17.3 & 5 & 0.004 & 71.0 & 0.02 & $-0.05-0.08$ & 0.482 & & \\
\hline Social support & 4 & 1057 & 2.8 & 3 & 0.423 & 0.0 & -0.24 & -0.29 to -0.18 & $<0.001$ & 49 & 30 \\
\hline${ }^{\wedge}$ Adjusted for publication bias ${ }^{f}$ & (6) & & & & & & $-0.26^{\wedge}$ & $-0.32^{\wedge}$ to -0.20 & & & \\
\hline \multicolumn{12}{|l|}{ Individual psychological- } \\
\hline \multicolumn{12}{|l|}{ behavioral predictors } \\
\hline Anxiety & 8 & 1552 & 39.1 & 7 & $<0.001$ & 82.1 & 0.07 & -0.01 to 0.15 & 0.080 & & \\
\hline Depression & 8 & 2018 & 48.1 & 7 & $<0.001$ & 85.4 & 0.07 & -0.01 to 0.15 & 0.106 & & \\
\hline \multicolumn{12}{|l|}{ Between-group analysesg } \\
\hline \multicolumn{12}{|l|}{ (psychological-behavioral states) } \\
\hline Generic pain measure & 3 & 310 & 1.8 & 3 & 0.623 & 0.0 & 0.10 & $-0.01-0.20$ & 0.061 & & \\
\hline Breast cancer-specific pain measure & 8 & 1866 & 31.6 & 7 & $<0.001$ & 77.8 & 0.04 & $-0.04-0.10$ & 0.354 & & \\
\hline ^Between-group & 11 & 2176 & $1.1^{\wedge}$ & $\left.\right|^{\wedge}$ & $0.290^{\wedge}$ & & & & & & \\
\hline Unadjusted analysis & 8 & 1636 & 9.6 & 7 & 0.210 & 27.4 & 0.09 & $0.03-0.15$ & 0.003 & 17 & 50 \\
\hline Adjusted analysis & 6 & 636 & 27.2 & 5 & $<0.001$ & 81.6 & 0.03 & $-0.04-0.10$ & 0.416 & & \\
\hline$\wedge^{\wedge}$ Between-group ${ }^{h}$ & $8^{\wedge}$ & $1488^{\wedge}$ & $0.9^{\wedge}$ & $\left.\right|^{\wedge}$ & $0.346^{\wedge}$ & & & & & & \\
\hline Presurgery baseline & 7 & 1338 & 17.8 & 6 & 0.007 & 66.4 & 0.08 & $0.01-0.14$ & 0.020 & 16 & 45 \\
\hline${ }^{\wedge}$ Adjusted for publication bias & & & & & & & $0.01^{\wedge}$ & $-0.05-0.06^{\wedge}$ & $\mathrm{ns}^{\wedge}$ & & \\
\hline Postsurgery baseline & 3 & 298 & 5.9 & 2 & 0.052 & 66.2 & 0.05 & $-0.14-0.24$ & 0.609 & & \\
\hline${ }^{\wedge}$ Between-group & $10^{\wedge}$ & $1636^{\wedge}$ & $0.07^{\wedge}$ & $I^{\wedge}$ & $0.794^{\wedge}$ & & & & & & \\
\hline Anxiety (HADS) & 3 & 291 & 2.3 & 2 & 0.313 & 13.9 & 0.10 & $0.05-0.15$ & $<0.001$ & 12 & 25 \\
\hline Anxiety (STAl) & 5 & 880 & 23.7 & 4 & $<0.001$ & 83.1 & 0.11 & $-0.00-0.21$ & 0.056 & & \\
\hline ^Between-group & $6^{\wedge}$ & $589^{\wedge}$ & $0.00^{\wedge}$ & $I^{\wedge}$ & $0.967^{\wedge}$ & & & & & & \\
\hline Depression (HADS) & 3 & 889 & 1.3 & 2 & 0.529 & 0.0 & 0.12 & $0.05-0.18$ & $<0.001$ & 5 & 25 \\
\hline Depression (BDI) & 3 & 191 & 2.7 & 2 & 0.260 & 25.9 & 0.03 & $-0.05-0.10$ & 0.437 & & \\
\hline ^Between-group & $6^{\wedge}$ & $1080^{\wedge}$ & $3.1^{\wedge}$ & $I^{\wedge}$ & $0.080^{\wedge}$ & & & & & & \\
\hline
\end{tabular}

Notes: ${ }^{a} Q$-statistic: $p$-values $<0.1$ taken to suggest heterogeneity. $P^{2}$ statistic: $0 \%$ (no heterogeneity), $25 \%$ (low heterogeneity), $50 \%$ (moderate heterogeneity), $75 \%$ (high heterogeneity). ${ }^{b}$ Fail-safe $n=$ number of nonsignificant studies that would bring the $p$ value to nonsignificant $(p>0.05)$. ${ }^{c} A$ fail-safe $n$ exceeding the criterion $(5 K+I 0)$ indicates a robust result. ${ }^{37 \mathrm{~d}} \mathrm{~K}=$ number of studies; $\mathrm{n}=$ total number of participants. ${ }^{\mathrm{E}} \mathrm{ESr}=$ effect size correlation. A positive value indicates an effect size in the hypothesized direction. All ESs were combined using a random effects model. Conventions: small (0.1); medium (0.3); large (0.5). ${ }^{33}$ ff analyses indicated possible publication bias, missing studies were imputed and an adjusted $\mathrm{ESr}$ calculated $\left({ }^{\wedge}\right){ }^{38}(\mathrm{~K})$ indicates number of published studies + number of imputed studies. ${ }^{8 B}$ Between-group analyses only conducted when $\mathrm{K}$ for each group $\geq 3$. ${ }^{h} \mathrm{~K}$ and $\mathrm{n}$ differ between individual analyses and comparisons, due to some studies presenting both adjusted and unadjusted results. Bold figure indicates a statistically significant result $(p<0.05)$.

Abbreviations: $d f$, degrees of freedom; ns, non-significant.

Table 4 Moderators of effects of psychosocial predictors of pain in women with breast cancer: results of meta-regression analyses $(\mathrm{K}>5)$

\begin{tabular}{|c|c|c|c|c|c|}
\hline Moderator & Predictor type & $\mathbf{K}^{\mathbf{a}}$ & Beta $^{b}$ & $95 \% \mathrm{Cl}$ & $p$-value (two-tailed) \\
\hline \multirow[t]{2}{*}{ Study quality } & Psychological-behavioral states & II & 0.020 & $-0.011-0.051$ & 0.198 \\
\hline & Psychological traits & 6 & -0.003 & $-0.047-0.041$ & 0.884 \\
\hline \multirow[t]{2}{*}{ Mean sample age (years) } & Psychological-behavioral states & II & 0.022 & $0.003-0.04 I$ & 0.023 \\
\hline & Psychological traits & 6 & -0.009 & $-0.037-0.020$ & 0.561 \\
\hline \multirow[t]{2}{*}{ Time to follow-up (months) } & Psychological-behavioral states & II & -0.005 & -0.007 to -0.002 & 0.0004 \\
\hline & Psychological traits & 6 & -0.003 & -0.005 to -0.0002 & 0.035 \\
\hline
\end{tabular}

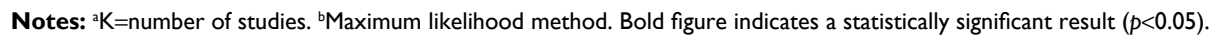

assessing social support did not focus on pain as the primary outcome, reported unadjusted analyses only, and assessed pain with generic quality of life pain subscales. The results thus need to be replicated using disease-specific pain measures and adjusting the results for relevant clinical variables.
However, the notion that social support reduces pain finds support in experimental studies with nonclinical populations ${ }^{53}$ and observational studies of chronic pain patients. ${ }^{54}$

Based on the previously published narrative reviews, 5,14 the lack of statistically significant associations between 
psychological-behavioral states and psychological traits and pain was unexpected. Based on the current conceptualization of pain as a multidimensional phenomenon including sensory as well as cognitive and emotional components, ${ }^{4,14}$ we expected that higher levels of negative psychological states or symptoms and potentially maladaptive traits (eg, alexithymia, trait anxiety) would predict higher levels of posttreatment pain. There are several possible explanations for our null findings. First, 10 out of 13 studies included pain as a categorical variable, thereby potentially reducing the amount of variance to be explained. Second, four studies had dichotomized pain as any pain vs no pain, failing to distinguish clinically significant from nonsignificant pain, thereby possibly masking the impact of psychosocial predictors on clinically relevant pain. Third, all studies focused on sensory pain in their statistical analyses, for example, pain intensity, and did not include evaluative or affective dimensions of pain, which could theoretically be more sensitive to psychological influences. ${ }^{55}$ Fourth, this review focused on breast cancer. Research in noncancer pain populations has found associations between anxiety, ${ }^{56}$ psychological symptoms, ${ }^{57}$ and depression ${ }^{58}$ and pain, and the impact of psychosocial predictors on pain may vary between different clinical populations. Finally, as suggested by meta-analyses, ${ }^{5,8,10}$ the variation in posttreatment pain in breast cancer may be mainly influenced by biomedical factors such as surgical and systemic treatments, body mass index, and preoperative pain, leaving only modest explanatory value to psychosocial factors. Although the difference did not reach statistical significance, our finding of larger effect sizes based on unadjusted analyses compared with those adjusted for clinical variables could be viewed as supportive of this explanation.

The effect sizes found for psychological-behavioral states and psychological traits appeared highly heterogeneous, which could be due to nonrandom differences, for example, in study characteristics. When addressing the possible moderating influence of a number of categorical and continuously assessed study characteristics, we found that longer time to follow-up was associated with smaller effect sizes and older sample age with larger effect sizes. While the first finding suggesting that the associations between psychological factors and pain diminish over time is less surprising, the latter is more unexpected as younger age has relatively consistently been identified as a risk factor for pain after breast cancer treatment. ${ }^{5,8,10}$ More research is needed to determine the moderating influence of age on the association between psychological factors and pain in breast cancer, when taking age-related differences, for example, menopausal status, in treatment into consideration.

The strengths of this systematic review include a comprehensive selection process with independent literature searches, study selections, and quality assessment. Furthermore, our review was preregistered and focused on prospective studies with increased likelihood of identifying causal associations. Finally, we quantified the predictive associations with meta-analytic strategies and examined heterogeneity, publication bias, and a number of possible moderators. Some limitations should also be noted. First, and most importantly, the number of available prospective studies was limited, potentially challenging the robustness of the findings. A post hoc power analysis suggests that the statistical power to detect the small pooled effect sizes found in the available research was limited. For example, for psychological-behavioral states, the power to detect a statistically significant pooled ESr of 0.05 with 11 studies with an average sample size of 198 was only $44 \%$. To detect an ESr of 0.05 with $80 \%$ statistical power would require at least 26 studies. The small number of studies also limited our ability to conduct relevant moderator analyses across the various psychosocial predictors. On a related note, we did not include gray literature, thereby possibly limiting the scope of this study. However, the search for gray literature is a less systematic process and gray literature studies are often of lower methodological quality, ${ }^{59}$ thereby compromising reproducibility and internal validity. We, therefore, chose to only include studies that were retrievable in electronic databases and instead address possible publication bias statistically. Second, the studies included in this meta-analysis were all conducted in Western countries, and as cultural differences has been suggested to exist in relation to pain, ${ }^{60}$ this clearly limits the cross-cultural generalizability of our findings.

\section{Conclusion}

Taken together, with the exception of social support, our findings did not support pretreatment psychosocial factors as predictors of breast cancer-related pain. This, however, does not exclude the clinical importance of psychosocial factors in rehabilitation, which is supported by studies showing that targeting psychosocial factors in pain rehabilitation is efficacious in reducing pain. ${ }^{61-63}$ Thus, psychosocial factors may still play an important role in the survivorship trajectory, and studies using designs that capture the dynamic and complex nature of the relationship between psychosocial factors and pain are needed. 


\section{Disclosure}

The authors report no conflicts of interest in this work.

\section{References}

1. Mejdahl M, Andersen K, Gärtner R, Kroman N, Kehlet H. Persistent pain and sensory disturbances after treatment for breast cancer: six year nationwide follow-up study. Br Med J. 2013;1865:1-14.

2. Gärtner R, Jensen M, Nielsen J, Ewertz M, Kroman N, Kehlet H. Prevalence of and factors associated with persistent pain following breast cancer surgery. J Am Med Assoc. 2009;302(18):1985-1992.

3. Johannsen M, Christensen S, Zachariae R, Jensen A. Socio-demographic, treatment-related, and health behavioral predictors of persistent pain 15 months and 7-9 years after surgery: a nationwide prospective study of women treated for primary breast cancer. Breast Cancer Res Treat. 2015;152(3):645-658.

4. The IASP Task Force on Taxonomy I. Part III: pain terms, a current list with definitions and notes on usage. In: Merskey H, Bogduk N, editors. Classification of Chronic Pain. 2nd ed. Seattle, WA: IASP Press; 1994:209-214.

5. Andersen KG, Kehlet H. Persistent pain after breast cancer treatment: a critical review of risk factors and strategies for prevention. $J$ Pain. 2011;12(7):725-746.

6. Hui D, Bruera E. A personalized approach to assessing and managing pain in patients with cancer. J Clin Oncol. 2014;32(16):1640-1646.

7. Aaronson NK, Mattioli V, Minton O, et al. Beyond treatment - psychosocial and behavioural issues in cancer survivorship research and practice. Eur J Cancer Suppl. 2014;12(1):54-64.

8. Wang L, Guyatt GH, Kennedy SA, et al. Predictors of persistent pain after breast cancer surgery: a systematic review and meta-analysis of observational studies. Can Med Assoc J. 2016;188(14):E352-E361.

9. Mejdahl MK, Mertz BG, Bidstrup PE. Preoperative distress predicts persistent pain after breast cancer treatment : a prospective cohort study. J Natl Compr Cancer Netw. 2015;13(8):995-1003.

10. Leysen L, Beckwée D, Nijs J, et al. Risk factors of pain in breast cancer survivors: a systematic review and meta-analysis. Support Care Cancer. 2017;25(12):3607-3643.

11. Novy DM, Aigner CJ. The biopsychosocial model in cancer pain. Curr Opin Support Palliat Care. 2014;8(2):117-123.

12. Melzack R, Wall P. Pain mechanisms: a new theory. Science. 1965;150(3699):971-979.

13. Melzack R. From the gate to the neuromatrix. Pain. 1999; Suppl 6(1): S121-S161.

14. Schreiber KL, Kehlet H, Belfer I, Edwards RR. Predicting, preventing and managing persistent pain after breast cancer surgery: the importance of psychosocial factors. Pain Manag. 2014;4(6):445-459.

15. Day MA, Jensen MP, Ehde DM, Thorn BE. Toward a theoretical model for mindfulness-based pain management. J Pain. 2014;15(7):691-703.

16. Belfer I, Schreiber KL, Shaffer JR, et al. Persistent postmastectomy pain in breast cancer survivors: analysis of clinical, demographic, and psychosocial factors. J Pain. 2013;14(10):1185-1195.

17. Schreiber KL, Martel MO, Shnol H, et al. Persistent pain in postmastectomy patients: comparison of psychophysical, medical, surgical, and psychosocial characteristics between patients with and without pain. Pain. 2013;154(5):660-668.

18. Bell RJ, Robinson PJ, Nazeem F, et al. Persistent breast pain 5 years after treatment of invasive breast cancer is largely unexplained by factors associated with treatment. J Cancer Surviv. 2014;8(1):1-8.

19. Miaskowski C, Cooper B, Paul SM, et al. Identification of patient subgroups and risk factors for persistent breast pain following breast cancer surgery. J Pain. 2012;13(12):1172-1187.

20. Katz J, Poleshuck EL, Andrus CH, et al. Risk factors for acute pain and its persistence following breast cancer surgery. Pain. 2005;119(1-3):16-25.

21. Bruce J, Thornton AJ, Powell R, et al. Psychological, surgical, and sociodemographic predictors of pain outcomes after breast cancer surgery: a population-based cohort study. Pain. 2014;155(2):232-243.
22. Meretoja TJ, Andersen KG, Bruce J, et al. Clinical prediction model and tool for assessing risk of persistent pain after breast cancer surgery. J Clin Oncol. 2017;35(15):1660-1667.

23. Poleschuk, Katz J, Andrus C, et al. Risk factors for chronic pain following breast cancer surgery: a prospective study. J Pain. 2006;7(9): 626-634.

24. Bishop SR, Warr D. Coping, catastrophizing and chronic pain in breast cancer. J Behav Med. 2003;26(3):265-281.

25. Andersen KG, Duriaud HM, Jensen HE, Kroman N, Kehlet H. Predictive factors for the development of persistent pain after breast cancer surgery. Pain. 2015;156(12):2413-2422.

26. Liberati A, Altman DG, Tetzlaff J, et al. The PRISMA statement for reporting systematic reviews and meta-analyses of studies that evaluate health care interventions: explanation and elaboration. JClin Epidemiol. 2009;62(10):34

27. Sackett DL, Rosenberg W, Gray J, Haynes J, Richardson W. Evidence based medicine: what it is and what it isn't. Br Med J. 1996;312(7023):71-72.

28. Landis JR, Koch GG. The measurement of observer agreement for categorical data. Biometrics. 1977;33(1):159-174.

29. McHugh M. Interrater reliability: the kappa statistic. Biochem Medica. 2012;22(3):276-282.

30. The National Institute of Health: National Heart Lung and Blood Institute (NILBI). Quality Assessment Tool for Observational Cohort and Cross-Sectional Studies. Available from:https://www.nhlbi.nih.gov/ health-pro/guidelines/in-develop/cardiovascular-risk-reduction/tools/ cohort. Accessed March 09, 2017.

31. Rosenthal R, Rubin DB. $r$ equivalent: a simple effect size indicator. Psychol Methods. 2003;8(4):492-496.

32. Lipsey MW, Wilson DB. The efficacy of psychological, educational, and behavioral treatment: confirmation from meta-analysis. Am Psychol. 1993;48(12):1181-1209.

33. Cohen J. Statistical Power Analysis for the Behavioral Sciences. 2nd ed. Mahwah, NJ: Lawrence Erlbaum Associates; 1988.

34. Ioannidis JPA, Trikalinos TA. The appropriateness of asymmetry tests for publication bias in meta-analyses: a large survey. Can Med Assoc J. 2007;176(8):1091-1096.

35. Egger M, Smith GD, Schneider M, Minder C. Bias in meta-analysis detected by a simple, graphical test. Br Med J. 1997;315(7109):629-634.

36. Copas J, Shi JQ. Meta-analysis, funnel plots and sensitivity analysis. Biostatistics. 2000;1(3):247-262.

37. Rosenthal R. The file drawer problem and tolerance for null results. Psychol Bull. 1979;86(3):638-641.

38. Duval S, Tweedie R. Trim and fill: a simple funnel-plot-based method of testing and adjusting for publication bias in meta-analysis. Biometrics. 2000;56(2):455-463.

39. Sterne J, Egger M, Moher D. Adressing reporting biases. In: Higgins J, Green S, editors. Cochrane Handbook for Systematic Reviews of Intervention. Chichester: Wiley-Blackwell; 2008:297-333.

40. Poole C, Greenland S. Random-effects meta-analyses are not always conservative. Am J Epidemiol. 1999;150(5):469-475.

41. Higgins JPT, Thompson SG, Deeks JJ, Altman DG. Measuring inconsistency in meta-analyses. BMJ. 2003;327(7414):557-560.

42. Dijkstra PU, Rietman JS, Geertzen JHB. Phantom breast sensations and phantom breast pain: a 2-year prospective study and a methodological analysis of literature. Eur J Pain. 2007;11(1):99-108.

43. van de Wiel H, Geerts E, Hoekstra-Weebers J. Explaining inconsistent results in cancer quality of life studies: the role of the stressresponse system. Psychooncology. 2008;17(2):174-181.

44. Hughes S, Jaremka LM, Alfano CM, et al. Social support predicts inflammation, pain, and depressive symptoms: longitudinal relationships among breast cancer survivors. Psychoneuroendocrinology. 2014;42:38-44.

45. Leung J, Pachana NA, Mclaughlin D. Social support and health-related quality of life in women with breast cancer : a longitudinal study. Psychooncology. 2014;23(9):1014-1020.

46. Meretoja TJ, Leidenius MHK, Tasmuth T, Sipilä R, Kalso E. Pain at 12 months after surgery for breast cancer. JAMA. 2014;311(1):90. 
47. Andersen KG, Duriad HM, Jensen HE, Kroman N, Kehlet H. Predictive factors for the development of persistent pain after breast cancer surgery. Pain. 2015;156(12):2413-2422.

48. Mejdahl M, Mertz B, Bidstrup P. Preoperative distress predicts persistent pain after breast cancer treatment: a prospective cohort study. $J$ Natl Compre Canc Netw. 2015;13(8):995-1003.

49. Baudic S, Jayr C, Albi-Feldzer A, et al. Effect of alexithymia and emotional repression on postsurgical pain in women with breast cancer: a prospective longitudinal 12-month study. J Pain. 2016;17(1):90-100.

50. Nijs J, Leysen L, Adriaenssens N, et al. Pain following cancer treatment: guidelines for the clinical classification of predominant neuropathic, nociceptive and central sensitization pain. 2016;55(6):659-663.

51. Martinez A, Zarazaga R, Maestre C. Perceived social support and coping responses are independent variables explaining pain adjustment among chronic pain patients. J Pain. 2008;9(4):373-379.

52. Kerns RD, Rosenberg R, Otis JD. Self-appraised problem solving and pain-relevant social support as predictors of the experience of chronic pain. Ann Behav Med. 2002;24(2):100-105.

53. Krahé C, Springer A, Weinman JA, Fotopoulou A. The social modulation of pain: others as predictive signals of salience - a systematic review. Front Hum Neurosci. 2013;7:1-21.

54. Jensen MP, Moore MR, Bockow TB, Ehde DM, Engel JM. Psychosocial factors and adjustment to chronic pain in persons with physical disabilities: a systematic review. Arch Phys Med Rehabil. 2011;92(1):146-160.

55. Geisser ME, Robinson ME, Keefe FJ, Weiner ML. Catastrophizing, depression and the sensory, affective and evaluative aspects of chronic pain. Pain. 1994;59(1):79-83.
56. Bair MJ, Poleshuck EL, Wu J, et al. Anxiety but not social stressors predict 12-month depression and pain severity. Clin J Pain. 2013;29(2): 95-101.

57. Lerman SF, Rudich Z, Brill S, Shalev H, Shahar G. Longitudinal associations between depression, anxiety, pain, and pain-related disability in chronic pain patients. Psychosom Med. 2015;77(3):333-341.

58. Bair MJ Katon W,Kroenke K. RRL. Depression and pain comorbidity: a literature review. Arch Intern Med. 2003;163(20):2433-2445.

59. Egger M, Jüni P, Bartlett C, Holenstein F, Sterne J. How important are comprehensive literature searches and the assessment of trial quality in systematic reviews? Empirical study. Health Technol Assess. 2003;7(1): $1-76$.

60. Peacock S, Patel S. Cultural influences on pain. Rev pain. 2008;1(2):6-9.

61. Johannsen M, Farver I, Beck N, Zachariae R. The efficacy of psychosocial intervention for pain in breast cancer patients and survivors: a systematic review and meta-analysis. Breast Cancer Res Treat. 2013;138(3):675-690.

62. Syrjala KL, Jensen MP, Mendoza ME, Yi JC, Fisher HM, Keefe FJ. Psychological and behavioral approaches to cancer pain management. J Clin Oncol. 2014;32(16):1703-1712.

63. Veehof MM, Oskam M-J, Schreurs KMG, Bohlmeijer ET. Acceptancebased interventions for the treatment of chronic pain: a systematic review and meta-analysis. Pain. 2011;152(3):533-542.

64. Laroche F, Coste J, Medkour T, et al. Classification of and risk factors for estrogen deprivation pain syndromes related to aromatase inhibitor treatments in women with breast cancer: A prospective multicenter cohort study. J Pain. 2014;15(3):293-303. 


\section{Supplementary material}

Table SI Full electronic search strategy and number of hits

\begin{tabular}{|c|c|c|c|c|}
\hline Search combination & PubMed $^{\mathrm{a}}$ & PsyclNFO $^{b}$ & Web of Science $^{c}$ & CINAHL $^{d}$ \\
\hline Search \#I & 236,763 & 9,820 & 207,559 & 20,624 \\
\hline \multicolumn{5}{|l|}{ "breast neoplasm" OR } \\
\hline \multicolumn{5}{|l|}{ "breast cancer" OR } \\
\hline \multicolumn{5}{|l|}{ "breast carcinoma" OR } \\
\hline \multicolumn{5}{|l|}{ "breast tumor" OR } \\
\hline \multicolumn{5}{|l|}{ "mammary cancer" } \\
\hline Search \#2 & 550,612 & 504,694 & $1,384,319$ & 248,872 \\
\hline \multicolumn{5}{|l|}{ Predict OR } \\
\hline \multicolumn{5}{|l|}{ Predictor OR } \\
\hline \multicolumn{5}{|l|}{ "risk factor" OR } \\
\hline \multicolumn{5}{|l|}{ "relative risk" OR } \\
\hline \multicolumn{5}{|l|}{ “odds ratio" OR } \\
\hline \multicolumn{5}{|l|}{ Logistic OR } \\
\hline \multicolumn{5}{|l|}{ Regression } \\
\hline Search \#3 & 519,885 & 79,200 & 491,274 & 86,238 \\
\hline \multicolumn{5}{|l|}{ Pain OR } \\
\hline \multicolumn{5}{|c|}{ "post mastectomy pain syndrome" OR } \\
\hline \multicolumn{5}{|c|}{ “persistent post surgical pain” OR } \\
\hline \multicolumn{5}{|c|}{ Ache OR } \\
\hline \multicolumn{5}{|c|}{ "chronic post surgical pain" } \\
\hline Search \# 4 & 278 & 138 & 295 & 226 \\
\hline \multicolumn{5}{|l|}{$\# I$ AND } \\
\hline \multicolumn{5}{|l|}{ \#2 AND } \\
\hline \#3 & & & & \\
\hline
\end{tabular}

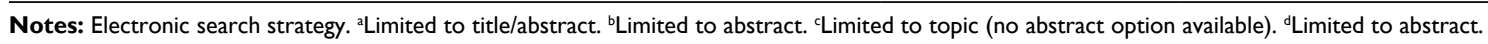

The Journal of Pain Research is an international, peer reviewed, open access, online journal that welcomes laboratory and clinical findings in the fields of pain research and the prevention and management of pain. Original research, reviews, symposium reports, hypothesis formation and commentaries are all considered for publication.
The manuscript management system is completely online and includes a very quick and fair peer-review system, which is all easy to use. Visit http://www.dovepress.com/testimonials.php to read real quotes from published authors. 